
italiana e internazionale dell'opera di Giorgio Bassani

\title{
«Un vero scrittore internazionale». La diffusione mondiale delle opere di Giorgio Bassani
}

"A True International Writer": The Worldwide Circulation of Giorgio Bassani's Works

"Un vrai écrivain international » : la diffusion mondiale des œuvres de Giorgio Bassani

Rosy Cupo

\section{OpenEdition}

\section{Journals}

Edizione digitale

URL: http://journals.openedition.org/cei/3785

DOI: 10.4000/cei.3785

ISSN: 2260-779X

Editore

UGA Éditions/Université Grenoble Alpes

\section{Edizione cartacea}

ISBN: 978-2-37747-040-2

ISSN: 1770-9571

Notizia bibliografica digitale

Rosy Cupo, ««Un vero scrittore internazionale». La diffusione mondiale delle opere di Giorgio Bassani», Cahiers d'études italiennes [Online], 26 | 2018, online dal 28 février 2018, consultato il 26 mars 2021. URL: http://journals.openedition.org/cei/3785 ; DOI: https://doi.org/10.4000/cei.3785

Questo documento è stato generato automaticamente il 26 mars 2021

(c) ELLUG 


\title{
«Un vero scrittore internazionale». La diffusione mondiale delle opere di Giorgio Bassani
}

\author{
"A True International Writer": The Worldwide Circulation of Giorgio Bassani's \\ Works \\ "Un vrai écrivain international » : la diffusion mondiale des œuvres de Giorgio \\ Bassani
}

Rosy Cupo

1 Nell'ambito delle numerose iniziative che hanno contribuito alle celebrazioni per il centenario della nascita di Giorgio Bassani, l'Università di Ferrara ha promosso una mostra incentrata sulla diffusione internazionale delle opere dello scrittore ferrarese. Il punto di osservazione prescelto, atto a ripercorrere e analizzare i modi, i tempi e le proporzioni di un fenomeno niente affatto secondario, si è rivelato poi importante per indurre una riflessione più ampia sulle ragioni letterarie ed extraletterarie di un successo mondiale. Il presente saggio si propone pertanto di approfondire i dati emersi da quella prima ricognizione ${ }^{1}$.

2 La ricerca ha preso avvio dallo studio dei carteggi intrattenuti da Giorgio Bassani con i principali esponenti del panorama culturale dell'Italia dell'immediato dopoguerra; a tal fine sono stati presi in esame i documenti dell'Archivio Giorgio Bassani di Parigi, quelli della casa editrice Einaudi, conservati presso l'Archivio Storico di Torino, e quelli dell'importante archivio dell'Agenzia Letteraria Internazionale, conservati presso la Fondazione Mondadori di Milano. La lettura sinottica dei materiali, consistenti per buona parte in lettere scambiate dallo scrittore con editori, agenti e traduttori di tutto il mondo, ha consentito di studiare le dinamiche dell'operazione editoriale (accordi preliminari, ricerca di un adeguato traduttore, promozione pubblicitaria dell'opera), e di ricostruire inoltre i rapporti con personaggi di rilievo del panorama culturale dell'epoca, come Erich Linder, Claude Gallimard, Giulio Einaudi e i suoi collaboratori: interlocutori privilegiati dello scrittore o chiamati in causa come semplici consiglieri 
(nel caso, ad esempio, di Italo Calvino), allorquando il giovane Bassani si trovò a muovere i primi passi nel campo letterario.

Uno dei risultati che il presente studio consegue è di mettere in evidenza l'impegno caparbio con cui Bassani ha sin dal principio perseguito l'obiettivo della pubblicazione all'estero; infatti i racconti di Bassani cominciano a essere tradotti subito, immediatamente dopo la pubblicazione in rivista, ben prima del successo delle Cinque storie. Si riscontra pertanto una volontà precisa, che non può essere esclusivamente ricondotta ad un mero movente economico, dal momento che le edizioni estere, come si evince chiaramente dai materiali analizzati, non procuravano che una piccolissima percentuale sulle vendite, e non rappresentavano certo fonte di arricchimento.

In tale operazione fu importante la mediazione di Marguerite Caetani, non tanto per i rapporti e i contatti già instaurati che poteva offrire al giovane redattore di «Botteghe oscure», quanto per l'impostazione mentale che infondeva al suo collaboratore affidandogli il compito di gestire una rivista che nasceva con una prepotente vocazione internazionale, prevedendo stabilmente, sin dal secondo numero, sezioni di poesia italiana, francese, inglese, statunitense e tedesca; ricordandola a poche settimane dalla sua scomparsa, Bassani affermava di aver appreso molto da lei, e in particolare le riconosceva il merito di avergli aperto gli occhi, in un momento in cui era ancora "portato a vivere una realtà soprattutto interiore e in un certo modo ossessiva», soprattutto sul piano letterario, e insegnandogli al tempo stesso a «prendersi meno sul serio» ${ }^{2}$.

5 La carriera di scrittore di Giorgio Bassani si avviò, come è noto, nel 1940 quando, giovanissimo, pubblicò la raccolta di racconti Una città di pianura sotto lo pseudonimo di Giacomo Marchi, a proprie spese, come ricorderà con commozione facendone dono a Giulio Einaudi nel gennaio del $1964^{3}$. Nel 1948 divenne redattore di «Botteghe oscure»: fu il primo quaderno di questa rivista a ospitare la novella Storia d'amore, seconda versione del racconto Storia di Debora, già pubblicato nella prima raccolta. La novella, ulteriormente rielaborata, diverrà la prima delle Cinque storie ferraresi, con il titolo Lida Mantovani. È stato già messo in evidenza lo strettissimo rapporto intercorso tra la nascita dei primi racconti e la cordiale sollecitazione, o «attesa [...] imperiosa» ${ }^{4}$, come Bassani stesso la definì, dell'amica Marguerite Caetani, che indusse lo scrittore a rielaborare la già citata Storia di Debora proprio per la pubblicazione su «Botteghe oscure». Ancora sotto il patrocinio della principessa Caetani, che della rivista era al contempo fondatrice e 'anima', si avviarono anche i primi tentativi, da parte di Bassani, di esportare all'estero la propria opera. L'esordio nel panorama letterario internazionale avverrà proprio con Love story in «An Anthology of New Italian Writers», una miscellanea, recita il sottotitolo, di autori le cui opere erano apparse sui numeri della rivista «Botteghe oscure» ${ }^{5}$. Subito dopo la pubblicazione su «Botteghe oscure» di Una passeggiata prima di cena (1951) la Caetani scrisse a Margaret Bottrall, scrittrice e studiosa americana, per affidarle la traduzione del racconto, come risulta da una lettera della Bottrall stessa del 23 luglio 1951, in cui la richiesta era approssimativamente fatta risalire a un mese prima; la traduttrice americana, pur apprezzando profondamente il racconto e la "tecnica sottile [con cui l'autore] evoca il tempo passato», non mancò di definire la traduzione «un'impresa assai difficile» soprattutto a causa di «tutte quelle parentesi». La traduzione fu approntata in pochi mesi di lavoro: possediamo infatti la minuta della lettera inviata da Bassani alla traduttrice ${ }^{6}$, in cui approvava il lavoro della Bottrall, definendolo «assolutamente magnifico: non solo essa riproduce il testo nei 
suoi significati particolari come meglio non avrebbe potuto desiderare, ma dà un equivalente efficacissimo del suo tono generale». Inoltre l'autore sottolineava «l'emozione [...] provata nuovamente a veder[si] tradotto nella lingua letteraria amata più al mondo...» ${ }^{7}$. Se non è facile stabilire quanto di ironico ci fosse nell'osservazione: «nel complesso, la traduzione migliora l'originale: nel senso che lo rende più chiaro, più limpido", è certo che pur in così precoci tentativi Bassani adottò un metodo che si manterrà sostanzialmente inalterato per l'intera sua carriera: la scelta ponderata $\mathrm{e}$ cauta del traduttore, l'attenta analisi dei risultati ottenuti, effettuata personalmente e con l'aiuto di un consulente, l'invio di proposte migliorative della traduzione. Nel caso in oggetto sarà il prof. Salvatore Rosati, anglista, americanista e anche lui collaboratore di «Botteghe oscure», a esaminare la traduzione insieme allo stesso Bassani, e a proporre mutamenti riguardanti l'aspetto grafico, lessicale e anche sintattico.

Nella medesima lettera Bassani incitava la Bottrall a ricercare personalmente una sede letteraria per collocarvi il racconto, in quanto Desmond Fitzgerald, direttore della rivista «World Review», preventivamente interpellato, aveva risposto evasivamente, preannunciando in ogni caso una lunga attesa dovuta al fatto che «troppo di recente [erano] apparse nella sua rivista cose italiane» ${ }^{8}$. Ciò causò un incidente diplomatico tra $\mathrm{i}$ due: Fitzgerald, rotto ogni indugio, aveva proceduto in maniera autonoma affidando a Gwyn Morris il lavoro di traduzione, per cui il lavoro della Bottrall andò perduto, nonostante il pronto coinvolgimento della Caetani, che tuttavia liquidò in un succinto poscritto il suo pensiero sulla questione: «Fitzgerald ha fatto tradurre suo racconto senza avvertire noi. È stupido e ridicolo la Bottrall è furiosa»" The Stroll before Supper uscì effettivamente sulla «World Review» nei primi mesi del 1952, e la traduzione Morris fu giudicata dalla Bottrall «buona ma meno fedele che la mia al testo originale» ${ }^{10}$.

7 Nessuna notizia certa è stato possibile rinvenire sulla effettiva realizzazione della proposta di includere un racconto di Giorgio Bassani in un'antologia di autori italiani a cura di un certo professor Marc Slonim per la casa editrice americana Simon \& Schuster, avanzata per il tramite di Ben Jonson ${ }^{11}$. Ma erano proprio questi gli anni in cui prendevano forma le Cinque storie ferraresi, date alle stampe nel 1956 nella versione «rivista e corretta» presso l'editore Einaudi. Già nel marzo gli accordi con la casa editrice erano stati siglati, e il 24 maggio lo scrittore ne stringeva in mano i primi esemplari, stampati con una tiratura totale di 3000 copie. L'edizione venne presumibilmente approntata in modo celere proprio per poter garantire la partecipazione al Premio Strega. Il carteggio intrattenuto in questi mesi febbrili con i diversi redattori della casa editrice Einaudi dimostrano il grande impegno e le non comuni doti organizzative dello scrittore, che pretese una distribuzione capillare e pronta, già in previsione della vittoria; così Armando Vitelli, redattore Einaudi, canzonava amichevolmente Bassani: «Vedo che oltre che un fine letterato sei anche un organizzatore e un attivista nato; singolare ambivalenza» ${ }^{12}$. La raccolta si aggiudicò il Premio Strega in quello stesso anno, ottenendo un grande successo, comprovato dalle ben tre ristampe che si succedettero in pochi mesi. La notizia della vittoria ebbe una grande risonanza: piovvero da ogni parte le lettere e i telegrammi recanti rallegramenti e congratulazioni per il premio ottenuto. Rinvigorito dall'importante riconoscimento, Bassani si mostrò ancor più esigente verso la casa editrice, con continui suggerimenti $\mathrm{e}$ lamentele neppure troppo velate, se echi di esse sono rinvenibili non solo nelle lettere di Bassani, conservate nell'Archivio della casa editrice Einaudi, ma anche in quelle di Vitelli e dello stesso Giulio Einaudi (che lo rassicurò personalmente sul fatto che «gli 
inconvenienti [...] lamentati sarebbero stati ridotti al minimo») ${ }^{13}$. Molto interessante la missiva con cui Armando Vitelli risponde alle sollecitazioni a far meglio dello scrittore, ma al contempo lo incita a proseguire sulla strada intrapresa, in cui sembrava aver rinvenuto la sua vena narrativa più congeniale e profonda: «Anche io sono d'accordo con Cecchi perchè tu continui a raccontare Ferrara. Ti è possibile? Ad ogni modo non far dire di te che sei quello dei due racconti e mezzo per lustro. Adesso hai una bella responsabilità ma sono certissimo che non la eluderai» ${ }^{14}$.

8 La pazienza dell'autore fu messa in realtà a dura prova, dal momento che diverse grandi città erano rimaste sguarnite di copie delle Cinque storie, sempre più richieste dopo lo Strega, soprattutto considerando che la metà della prima tiratura era già stata venduta ancor prima che il Premio giungesse. Pertanto, neppure le rassicurazioni dello stesso Giulio Einaudi soddisfecero le aspettative dell'autore, se in una cartolina datata 8 gennaio 1957 scriveva ancora a Vitelli: «Bada, sto scrivendo un romanzo che sarà quasi certamente un capolavoro [...]. Ma se non vedrò che trattiate un po' meglio $i$ vostri ed i miei interessi, sarò perfino capace di venderlo a Garzanti» ${ }^{15}$ : è certamente questo un cenno dell'autore al cantiere del suo primo romanzo, Gli occhiali d'oro.

Come è già emerso abbastanza chiaramente, Bassani fu un uomo assai energico $e$ intraprendente; parimenti rapida e incalzante, quindi, fu la campagna per la diffusione all'estero; evidentemente l'autore aveva investito molto nella concreta aspettativa che lo Strega agisse come cassa di risonanza anche fuori d'Italia. La più antica richiesta attestata, pervenuta a Bassani già nel luglio del 1956, proveniva dalla Svezia, nello specifico dalla traduttrice e agente letteraria Karin De Laval, la quale aveva scritto direttamente all'autore chiedendogli di premere affinché l'incarico della traduzione del volume le fosse affidato; Luciano Foà rispose a Bassani spiegando che il più grande limite dato dalla collaborazione della De Laval consisteva nel fatto di «vincolare il collocamento dei diritti al fatto che la traduzione fosse affidata a lei», confondendo quindi «il mestiere di agente letterario con quello di traduttrice»; considerazione che aveva indotto la Einaudi ad affidare la rappresentanza esclusiva a un'altra agente letteraria svedese, Karin Alin. Allo stato attuale delle nostre ricerche, tuttavia, pare che le Cinque storie ferraresi non siano mai state pubblicate in Svezia.

Molto più concreto dovette sembrare a Bassani il progetto di pubblicazione negli Stati Uniti, per il quale si adoperò energicamente e a lungo. La traduzione di uno dei racconti, Una notte del '43, era stata approntata da Raymond Rosenthal, già collaboratore di «Botteghe oscure», «per suo conto», come sostiene lo stesso Bassani ${ }^{16}$, ma certo con desiderio di autopromozione, e non senza il consenso (o l'incitamento) della Caetani; quest'ultima aveva interpellato Blanche Knopf, presidentessa della casa editrice statunitense Alfred A. Knopf Inc. (fondata nel 1915 con il marito Alfred Knopf) con la quale intratteneva una stretta amicizia; dopo meno di un mese, Bassani scriveva a Foà:

Pare che Knopf, l'editore americano, abbia intenzione di prendere il mio libro.

Vorrebbe però che io gli procurassi il volume già tradotto. [...] L'amico traduttore disposto a ciò (ha già tradotto per suo conto, e benissimo, Una notte del '43) si chiama Raymond Rosenthal e vive a Roma. Il suo nome è perfettamente gradito anche a Knopf $^{17}$.

11 Del tutto comprensibili le perplessità di Foà, il quale fece notare a Bassani come fosse un «modo di comportarsi singolare» chiedere di far eseguire l'intera traduzione prima di decidere se pubblicarlo o meno, soprattutto considerata l'esistenza della traduzione già approntata di un intero racconto. Ma non è peregrina l'ipotesi che Bassani, volitivo com'era e profondamente consapevole del valore della propria opera, abbia in questa 
occasione cercato di forzare un po' gli eventi. I punti salienti della vicenda sono presto riassunti: in un primo momento una lettera ricevuta dalla segretaria della Knopf, Eleanor French, informò Bassani che l'editrice aveva ricevuto una copia delle Cinque storie inviata da Curtis Brown (agente letterario della Einaudi operante negli Stati Uniti) per iniziativa di Erich Linder, e che sarebbe stata «most happy to see the translation by Mr. Raymond Rosenthal of the last story, A Night in '43»"18; a ciò seguì un promettente incontro a Parigi. Ma la risposta definitiva, giunta in tempi relativamente brevi, era stata, inaspettatamente, assai «scoraggiante»" ${ }^{19}$, come eufemisticamente la definì la Caetani, inviando contestualmente a Bassani la lettera recante il parere della Knopf, che qui si riporta per intero data la sua rilevanza:

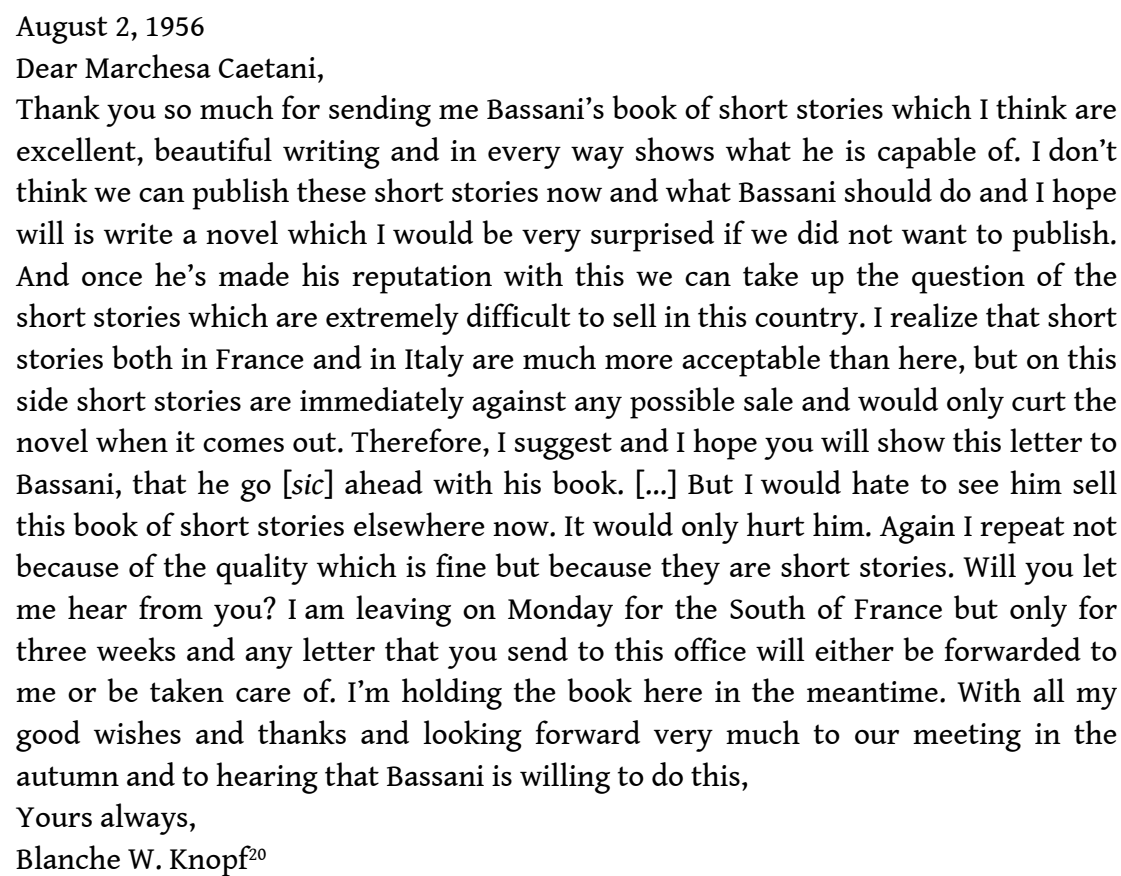

12 Come si può leggere, la decisione della Knopf, per quanto estremamente gentile e poco formale, si presentava come inappellabile. Incrociando le testimonianze giunteci, si spiega altresì come Bassani non potesse rassegnarsi facilmente di fronte a una valutazione così favorevole nell'insieme, che tuttavia non si risolveva felicemente; $\mathrm{e}$ forse ritenne di poter vincere la ritrosia dell'editrice inviando la traduzione dell'opera completa, $\mathrm{o}$, ancora, che una traduzione completa già approntata potesse accelerare l'accordo con un "altro editore americano» ${ }^{21}$. Un anno più tardi, un nuovo incontro con la Knopf faceva rinascere le speranza in Bassani, che scrisse alla Caetani: «Mi ha scritto da Parigi Blanche Knopf, immagino per suo suggerimento. Grazie di cuore. L'importate signora sarà qui l'11, e ci resterà fino al 17. L'attendo a piè fermo, con la traduzione di Rosenthal in mano» ${ }^{22}$.

13 Ma l'intraprendenza di Bassani non si limitava a cercare di sfruttare al massimo le occasioni che gli si presentavano: egli lavorava alacremente anche per crearne di nuove; di fronte all'ennesima sollecitazione riguardante le Cinque storie, Foà rispondeva: «finora nessuna offerta ci è pervenuta. Con gli editori esteri bisogna armarsi di pazienza; è molto difficile che un libro sia collocato entro tre o quattro mesi dalla pubblicazione $»^{23}$. Ma, nonostante il risoluto responso proferito, un suggerimento era giunto proprio dalla editrice americana Knopf, che aveva notato come le «short stories both in France and Italy are much more acceptable than here»; sicuramente grazie al 
suo interessamento, infatti, Bassani contattò Mrs. Bradley, amica della suddetta e agente letteraria residente in Francia. Bassani incontrò a Roma le due donne, informandone subito la Caetani: «Ho consegnato alla signora Bradley, che la leggerà subito, la traduzione del mio libro fatta da Rosenthal. Tanto la Bradley che la Knopf mi sembrano molto ben disposte nei miei riguardi. Speriamo bene» ${ }^{24}$.

Proprio per cercare di agevolare il più possibile questa seconda possibilità, nello stesso periodo Bassani scrisse per la prima volta direttamente a Erich Linder, direttore dell'Agenzia Letteraria Internazionale ${ }^{25} \mathrm{e}$ agente di Einaudi per i diritti di traduzione all'estero, trovando infatti opportuno comunicargli di aver affidato, «sia pure verbalmente [...] l'incarico [...] di fare pubblicare il libro in Francia e in Inghilterra» ${ }^{26}$ alla signora Bradley. Il rapporto si avviò nel segno di una forte diffidenza, dovuta al fatto che Bassani non aveva mai ricevuto prima d'allora nessuna offerta; le parole piuttosto dure inizialmente impiegate ${ }^{27}$, vennero tuttavia dimenticate non appena l'autore poté, approfondendone la conoscenza, saggiare la competenza del direttore dell'ALI, soprattutto in situazioni delicate: il carteggio fa risaltare appieno la potente personalità, la fermezza del carattere, la professionalità di Erich Linder, grazie alle quali egli riuscì in breve tempo ad accaparrarsi la completa fiducia di uno scrittore pur esigente come Giorgio Bassani.

15 L'analisi commerciale compiuta da Mrs. Knopf era sostanzialmente corretta: se le Cinque storie ferraresi erano state frenate, nella loro diffusione, dal genere novellistico, commercialmente poco appetibile, cui appartenevano, pure avevano creato una forte attesa tra gli specialisti del settore; ecco perché la pubblicazione del nuovo romanzo, Gli occhiali d'oro, uscito sempre presso Einaudi nei primi mesi del 1958, suscitò immediatamente una pioggia di richieste di pubblicazione all'estero. Il primo ad attivarsi fu il prestigioso editore francese Gallimard, che inviò una proposta a Bassani battendo di poco sul tempo un altro editore francese, Les Éditions du Seuil. Ciò comportò per l'autore la difficoltà di scegliere tra i due, aiutato però da un consigliere d'eccezione, che parteggiava per Seuil: Italo Calvino ${ }^{28}$. A quest'ultimo Bassani scrisse una cartolina che dimostra quanto tenesse al parere dell'amico, sebbene, come dirà apertamente in una successiva lettera a Foà, preferisse Gallimard per il fatto che garantiva alle opere «una circolazione praticamente mondiale» ${ }^{29}$. Nella stessa lettera Bassani pone, quasi colto da un presentimento, una questione fondamentale, quella della ricerca del traduttore migliore: «Sono convinto che il successo del libro sia legato strettamente a una buona traduzione $\aleph^{30}$. Ancora nell'agosto del ' 58 giunsero le proposte dell'editore inglese Faber \& Faber e dell'americano Harcourt Brace, seguite nel dicembre dal tedesco Piper. Bassani rispose con entusiasmo:

Sono al settimo cielo dalla felicità. Le tue notizie, che arrivano una dopo l'altra, mi danno la sensazione di essere un vero scrittore internazionale. [...] Sono assolutamente d'accordo circa la Harcourt Brace di New York. Ho avuto anche un'altra proposta, tramite Raymond Rosenthal, che, come sai, ha già tradotto le Cinque storie ferraresi, dall'editore McGraw Hill ${ }^{31}$.

Il romanzo Gli occhiali d'oro fu la prima opera di Bassani pubblicata all'estero: in Gran Bretagna e negli Stati Uniti nella versione di Isabel Quigly nel 1960, nello stesso anno in Germania tradotta da Herbert Schlüter. Ma la firma del contratto (Bassani l'avrebbe presto constatato) non significava affatto una pronta e soddisfacente conclusione del percorso editoriale. Due questioni in particolare, come emerge dall'analisi dei carteggi, si trascinarono a lungo: la pubblicazione del volume francese Les lunettes d'or et autres 
histoires de Ferrare ${ }^{32}$ presso l'editore francese Gallimard, e quella di The Garden of the Finzi-Continis per la Atheneum Publishers di New York.

Per l'edizione francese Bassani aveva immediatamente scritto a Paul-Henry Michel, sperando che accettasse di incaricarsi della traduzione. Dinanzi al rifiuto di quest'ultimo, Gallimard incaricò una certa Madame Mireille Rose (che Bassani si ostinò sempre, probabilmente con ironia, a chiamare Rosé); ma la lettura della traduzione gelò lo scrittore: questa risultava «inaccettabile». Linder venne immediatamente messo al corrente della questione:

Colgo l'occasione per dirle che ho rifiutato la traduzione francese delle Storie ferraresi, che avrebbero dovuto, come sa, uscire da Gallimard. La traduzione era semplicemente infame, una vera e propria falsificazione, tale che, se fosse uscita, mi avrebbe precluso ogni futura possibilità in Francia. [...] La traduttrice del mio [libro], madame Mireille Rosé, è una brava signora che prima d'ora non aveva mai fatto nessun lavoro del genere. Naturalmente, messa a confronto con un testo di quella difficoltà, ha combinato un incredibile pasticcio. [...] Tengo ad avvertirla che sono deciso a fare qualsiasi cosa per impedire che le Storie ferraresi vengano fuori in simile veste ${ }^{33}$.

Allegata alla lettera sopra riportata si trova, conservata oggi nell'Archivio dell'ALI, anche una copia della lunghissima lettera inviata a Michel Mohrt, redattore della casa editrice Gallimard; in essa Bassani motiva il rifiuto della traduzione, illustrando dettagliatamente i grossolani errori compiuti dalla «femme charmante, qui de sa vie n'a traduit un livre». La lettera rappresenta un documento di eccezionale importanza, sia per le informazioni che adduce sulla delicata operazione di traduzione, sia perché in essa Bassani compie, servendosi del confronto con il testo in oggetto, un'interessantissima analisi delle caratteristiche della propria prosa, definita «lyrique» ma al contempo «fort logique». Il primo e più grave problema che la traduzione presentava era rappresentato dall'indebito passaggio dal discorso indiretto libero al discorso diretto, su cui l'autore si sofferma lungamente, spiegando la peculiarità e l'importanza del primo all'interno del proprio stile e i riverberi sull'interpretazione stessa della realtà affidata ai propri racconti:

Il s'agit là d'une caractéristique fondamentale de mon style, caractéristique qui me permet de donner une représentation de la réalité extrêmement nuancée et ductile. De cette façon, le dialogue est presque toujours évité, afin, précisément, de mieux sauvegarder le réalisme. Le narrateur tient, dès la première ligne, tous les fils du récit, et ne veut surprendre le lecteur que par une interprétation à la fois critique et lyrique des faits. Le discours indirect s'étend et se développe parfois le long de plusieurs pages, quand il ne s'agit pas d'entiers chapitres. À la rigueur pourrait-on soutenir que tout le livre, tous les récits ne sont que de long discours indirects, où les rares dialogues constituent seulement des citations fortuites, qui servent à épauler les perpétuels raisonnements sur les sentiments et les choses.

Non siamo in possesso del testo originale di Madame Rose, ma Bassani inserisce una corposa serie di esemplificazioni, che ci consentono di verificare, in parte, le sue osservazioni:

1) Lida Mantovani, p. 41 (traduction):

« Je sais bien, semblait-il vouloir dire, qui est le père de cet enfant ... etc.

À mon avis, il aurait fallu traduire :

Oui, lui le savait parfaitement, semblait-il vouloir dire, qui avait été le père de cet enfant. Il savait tout. Il croyait à la sincerité de la passion juvénile qui l'avait poussée à se donner, à se perdre, il connaissait la douleur qui lui en était venue ... etc. 

d'une manière plus profonde et continue fausse les perspectives des temps» e il ritmo della frase, ottenuto attraverso un sapiente uso della punteggiatura e della paragrafatura, oltre che dei tempi verbali: «l'un des traits caractéristiques de ma prose est le sens du rythme. M.me Rosé a mis sens dessus dessous chaque page, n'y respectant ni la ponctuation ni surtout les alinéas»; il mancato rispetto di tali caratteristiche del testo originale da parte di «M.me Rosé» ha comportato un appiattimento dei piani temporali, conseguendo un risultato «catastrophique», e un testo «tassé comme de la chair à saucisse». In ultimo, l'autore segnala anche dei veri e propri fraintendimenti lessicali, e le intromissioni della traduttrice, che di propria iniziativa, considerando l'autore troppo «pédant, prolixe et ennuyeux, [...] abrège, bouleverse, allonge, au point d'oublier parfois l'original !».

Per quanto Bassani fosse un uomo esigente e fors'anche difficile da trattare, pure le sue osservazioni, qui riassunte, non appaiono eccessive o peregrine. Ciononostante, il confronto sfociò nell'aperta polemica, come si può leggere nella risposta di Michel Mohrt, conservata nell'archivio dell'ALI di Milano (e quindi inviata per conoscenza a Erich Linder, ormai consigliere fidato di Bassani); la lettera di Mohrt, quasi altrettanto lunga e circonstanziata, da un lato ricusa di aver imposto la Rose a Bassani, e riconosce che «Madame Rose a pris avec votre texte des libertés parfois trop grandes; notamment je la blâme d'avoir remplacé le discours indirect par un discours direct» e che «il y a des cas où Madame Rose aurait pu obtenir le même résultat sans altérer autant votre texte»; dall'altra, però, giustifica l'accaduto forse con un po' troppa indulgenza, sostenendo che "comme il vous arrive quelquefois à vous-même d'introduire dans le discours indirect quelques phrases en discours direct, elle s'est crue autorisée sans doute à en ajouter», e che la traduzione è, nonostante tutto, «écrite dans un français excellent»; Mohrt rifiuta inoltre decisamente il saggio di traduzione di Pressac inviatogli contestualmente da Bassani, definendolo un «mot à mot absolument impubliable, et la plupart du temps illisible». Ancora più interessante il prosieguo della lettera, in cui il redattore di Gallimard amplia il discorso fino a porre una questione generale, riguardante l'«arte del tradurre»:

Le lecteur français ne va pas faire ce que vous faites d'une façon inlassable, c'est-àdire confronter le texte original et la version française. Ce que demande le lecteur français, c'est un texte agréable à lire, qui ne sent pas la traduction. Autrement dit, le traducteur doit interpréter son texte pour en donner une autre version, conforme au génie d'une autre langue que la langue originale. Cela ne peut pas être obtenu par le mot à mot. [...] La fidélité n'est qu'un des éléments d'une bonne traduction, et sans doute pas le plus important. Le plus important, c'est que le texte de la traduction soit agréable à lire.

La proposta di Mohrt consisteva, in definitiva, nel correggere il testo della Rose, magari con l'aiuto di Bassani stesso, che si prendesse «la peine de lui signaler trois ou quatre exemples en lui disant dans quel sens vous souhaitez qu'elle travaille», mentre Bassani aveva più volte ribadito la propria irremovibile opposizione alla traduzione Rose. Pur adottando tutte le precauzioni del caso, e senza alcuna intenzione di elevare questo episodio a caso tipico, la vicenda può essere d'aiuto nell'analisi dei rapporti tra Bassani e la casa editrice Gallimard, e indurre, al contempo, una riflessione sulla politica editoriale che Gallimard adottò nei confronti delle Cinque storie ferraresi. Se da un lato può apparire fondata l'ipotesi avanzata di Vincent Raynaud ${ }^{34}$, che la difficile collocazione ideologica di Bassani lo rendesse un autore non particolarmente gradito, è 
pur vero, e le lettere lo testimoniano, che Bassani fu letteralmente "corteggiato» dall'editore francese; non si può poi non paragonare tale modo di procedere con quello di un'altra casa editrice, come la Barral Editores, poi Seix Barral, che facendo affidamento proprio sulla limitatezza della lettura critica dei censori, che non consentiva loro di cogliere il carattere nuovo, quasi eversivo delle opere di Bassani, tentò di aggirarla, perseverando a lungo nel tentativo di ottenere il nulla osta alla pubblicazione ${ }^{35}$. È quindi comprensibile e forse addirittura condivisibile la percezione, che Bassani certamente ne derivò, di essere stato ridotto, lui che si definiva «il contrario di un autore naturalista, (di uno scrittore, cioè, che crede alla realtà oggettiva)», a «una specie di autore di romanzi d'appendice!». La vicenda qui brevemente riassunta comportò ovviamente un ritardo nella pubblicazione del volume, che infatti uscì solo nel 1962, tradotto da Michel Arnaud ${ }^{36}$.

Con la data impressa del 9 febbraio 1962 uscì per la casa editrice Einaudi Il giardino dei Finzi-Contini. Il romanzo riscosse immediatamente un enorme successo, vendendo centomila copie in cinque mesi e aggiudicandosi l'importante riconoscimento letterario «Premio Viareggio». Nell'aprile dello stesso anno Giorgio Bassani volò negli Stati Uniti dove avrebbe dovuto discutere le condizioni della pubblicazione con Atheneum Publisher; l'accordo fu prontamente raggiunto, come dimostra un telegramma entusiasta inviato congiuntamente da Bassani e Simon Bessie da New York: «Talked today all agreed regards / Bassani Bessie» ${ }^{37}$. Ma ancora una volta approntare una degna traduzione del romanzo si rivelò un ostacolo quasi insormontabile. Il giardino dei FinziContini era già stato tradotto in Inghilterra per Faber \& Faber da Isabel Quigly, ma la traduzione, si evince da una lettera di Simon Bessie, dopo una lunga consultazione che aveva coinvolto Bassani, Linder e lo stesso Bessie, non era stata considerata adeguata, anche se, in questo caso, non siamo in possesso di informazioni dirette sui difetti riscontrati nella traduzione. Sin da subito viene proposto il nome di William Weaver come nuovo traduttore, ma purtroppo questi, che era legato da lunga e profonda amicizia a Bassani, non poté accettare a causa di impegni presi precedentemente. Dopo un lungo periodo di incertezza e dubbi, finalmente la traduzione Quigly fu sottoposta alla revisione di Cornelia Schaefer, che la trovò buona: le correzioni apportate si concentravano tutte nelle prime cento pagine, segno che, come scrive Linder, «la signorina Quigly aveva trovato una più equilibrata misura nel tradurre, con il procedere del lavoro». L'opinione di Linder, presumibilmente condivisa da Bassani, è che «il rischio più forte», ossia di «una traduzione manchevole» che impedisse di apprezzare l'opera, fosse scongiurato, e che la versione inglese avesse «nei limiti del possibile, tutta la precisione e tutte le necessità stilistiche dell'originale»: fu pertanto deciso di accettare la traduzione Quigly. Il 28 maggio del 1965, Bassani ne ricevette la prima copia. L'edizione americana ottenne molte recensioni positive, come dimostrano le numerose lettere inviate sia tramite Linder che dall'editore stesso.

Ma ormai la fama dello scrittore aveva travalicato i confini nazionali: Il giardino dei FinziContini venne pubblicato anche in Svezia, presso l'editore Albert Bonniers di Stoccolma, Danimarca, Norvegia, Portogallo, Olanda, Israele. Molto interessante la lettera in cui Bassani, in una lettera indirizzata a Abe Runnquist della casa editrice svedese, critica la scelta del titolo Il giardino perduto, per il fatto che «assomiglia troppo al "Tempo perduto" di Proust, ed io, nonostante le apparenze, non sono affatto proustiano». Non tardò la risposta in cui l'editore sottolineava che «il titolo svedese non riconduce all'opera di Proust, in quanto la parola "förlorad" [perduto] non è utilizzata nel titolo 
svedese dell'opera proustiana». Nell'autunno dello stesso anno Bassani compì un lungo viaggio nei paesi del nord, per promuovere il suo romanzo e tenere un ciclo di conferenze e letture.

Più difficile la penetrazione delle opere di Bassani nei territori di area comunista. Nonostante ciò, Il giardino dei Finzi-Contini fu pubblicato in Ungheria e in Polonia; del lavoro di traduzione in lingua polacca rimane una preziosa testimonianza nella lettera della traduttrice Jadniga Gaturska, che nel marzo del 1964, pochi mesi prima dell'uscita del libro, scrive direttamente all'autore per domandargli alcuni chiarimenti utili alla revisione finale. L'eco del successo delle opere di Giorgio Bassani giunge addirittura in Russia, anche se tardivamente, nel 1970: lo scrittore riceve una proposta di traduzione riguardante sia Il giardino che Gli occhiali d'oro. Ma i progetti avanzati rimasero tali, e l'unica opera bassaniana pubblicata nell'ex Unione sovietica rimase a lungo il racconto Una notte del '43, uscito con la traduzione di Lev Verscinin in una antologia di autori italiani del XX secolo ${ }^{38}$.

Nel 1964 fu pubblicato, sempre presso Einaudi, il nuovo romanzo Dietro la porta. Il successo mondiale era ormai consolidato, e i rapporti con le case editrici estere già stretti: non si trattava che di rivedere le condizioni contrattuali e procedere con la traduzione. Mentre il traduttore storico e unico delle opere di Bassani in Germania, Herbert Schlüter, appronterà anche la traduzione di Dietro la porta, Michel Arnaud, il traduttore francese, rifiuterà, perché sobbarcato dal lavoro, di portare a termine quest'altra impresa; ma sarà lui stesso a suggerire come nuovo traduttore Gérard Genot, studioso di lingua e cultura italiana e traduttore anche della Giornata di uno scrutatore, di Italo Calvino.

Nel 1968 Giorgio Bassani diede alle stampe per i tipi di Mondadori L'airone, ultimo romanzo, insignito l'anno successivo del prestigioso premio letterario «Campiello», messo in palio dagli industriali veneti. Anche questo romanzo ebbe diffusione mondiale, forse anche in misura maggiore rispetto ai precedenti; nello stesso anno Bassani dovette nuovamente affrontare la spinosa questione di una nuova traduzione inglese del Giardino dei Finzi-Contini; le lettere dell'archivio dell'ALI giacenti presso la Fondazione Alberto e Arnoldo Mondadori di Milano ci permettono di ricostruire, in parte, la vicenda: scartata nuovamente l'ipotesi di attendere che William Weaver si dedicasse al lavoro di traduzione, Giorgio Bassani procurò diversi saggi di traduzione, affidati a un tale Melchiori (un appunto di mano di Bassani reca «B. Melchiori»; ma si tratta probabilmente di Giorgio Melchiori, un traduttore allievo di Mario Praz) e a Vivian Wolbach, «ex moglie di Mario Praz», entrambi considerati insoddisfacenti. Finalmente lo scrittore si arrese all'idea di attendere la traduzione di Weaver, già traduttore de Gli occhiali d'oro nel 1963, e a cui si deve anche la prima edizione americana delle Storie Ferraresi, con il titolo Five stories of Ferrara (1971), presso la casa editrice Harcourt Brace Jovanovich di New York.

L'ultima opera pubblicata vivente l'autore è L'odore del fieno, una raccolta di racconti uscita per Mondadori nel 1972. Contemporaneamente maturò nell'autore il desiderio e quasi l'urgenza di procedere ad una profonda revisione formale di tutte le proprie opere; la seconda edizione delle Cinque storie ferraresi, intitolata Dentro le mura, fu riunita, insieme ai quattro romanzi e all'ultima raccolta di racconti, sotto un unico titolo: Il romanzo di Ferrara. Anche questa edizione completa ha conosciuto una buona diffusione mondiale. 

essa il direttore dell'ALI riferiva di una discussione intrattenuta con Helen Wolff (editor della Harcourt Brace Jovanovich) incentrata sul piano editoriale delle opere di Giorgio Bassani negli Stati Uniti: secondo Linder la casa editrice Harcourt Brace si era impegnata a creare una sicura piattaforma per la diffusione delle opere di Giorgio Bassani negli Stati Uniti, che avrebbe potuto essere resa «più fragile» dalla pubblicazione di una seconda raccolta di romanzi, cioè L'odore del fieno, dopo Dietro la porta. A distanza di quasi vent'anni, Bassani si vide nuovamente sbarrare la strada dalla «ragione (non valida letterariamente ma valida, purtroppo, negli Stati Uniti sul piano commerciale) che si tratta di un libro di racconti». La strategia commerciale proposta consisteva quindi nell'«attendere il nuovo romanzo, che [la Wolff] pubblicherebbe subito, e a cui farebbe seguire L'Odore del fieno e subito dopo, Gli occhiali d'oro [ristampa]». Ma forse Bassani sapeva in cuor suo che «il nuovo romanzo» non sarebbe più stato scritto.

30 Comparando le dinamiche di diffusione de L'odore del fieno con quella delle Cinque storie ferraresi, si nota in effetti una notevole somiglianza; anche L'odore del fieno fu tradotto in tempi brevi nei principali paesi Europei: immediatamente in Inghilterra, nel 1974 in Spagna e Germania, nel 1979 in Francia; ma sbarcherà negli Stati Uniti solo con la nuova traduzione (la terza in lingua inglese) promossa dall'importante casa editrice Penguin e curata dal poeta e scrittore inglese Jamie McKendrick, avviatasi nel 2007 con The Garden of the Finzi-Continis.

31 Nel centenario della nascita, la fama dello scrittore ferrarese continua a crescere. Anche la sua produzione poetica viene oggi riletta e studiata con rinnovato interesse, e tradotta in nuovi paesi, come dimostra la pubblicazione nel 2008 di $Z$ rimo in brez, la traduzione in lingua slovena di una scelta di liriche bassaniane; e quella, avvenuta per la prima volta nel 2014 in Cina, del Giardino dei Finzi-Contini (ma anche in Russia, come si è visto, e ancora in Thailandia e in Corea). Nel ripercorrere questa appassionante storia, i carteggi si rivelano una fonte preziosa di informazioni e consentono, come si è visto, di seguire da vicino le dinamiche dell'operazione editoriale, delineando la fisionomia di un autore estremamente esigente, intento a sorvegliare personalmente l'intero processo al fine di scongiurare il pericolo che la trasposizione linguistica potesse tradire l'opera, o che la letterarietà del testo venisse subordinata a una presunta «leggibilità».

fir studi bassaniani, in quanto affronta, in maniera neanche troppo indiretta, il tema del «successo» delle opere di Bassani, e induce ad interrogarsi sulle cause di esso; allo stesso tempo sfiora la questione, ancora aperta, della valutazione critica non sempre positiva (per usare un eufemismo) a lui contemporanea. Il successo delle opere bassaniane è stato infatti costantemente imputato all'autore come la misura e in qualche modo il segno più evidente del carattere «popolare» della sua scrittura. In una intervista pubblicata sul «Corriere della Sera» nel $1964^{39}$, pochi giorni prima dell'uscita di Dietro la porta, Bassani si sentiva porre questa capziosa domanda: «Credete che il successo del vostro romanzo [Il giardino dei Finzi-Contini] abbia delle ragioni che vanno al di là, per così dire, dell'esempio che rappresenta?»: il giornalista insinuava così pesantemente che il successo del romanzo fosse sproporzionato rispetto al valore del romanzo stesso, e da attribuirsi a ragioni estrinseche. Ma Bassani elegantemente rispondeva: «È fuor di dubbio, certissimo, che il successo del Giardino ha dietro ragioni 
che vanno oltre il mio "merito personale". Io valgo molto meno del Giardino dei FinziContini».

Ma tra le ragioni del successo estero delle opere di Giorgio Bassani è, a parer mio, senz'altro da annoverare l'innata e per niente scontata apertura mentale di un autore che si rivolge sin dal principio, intenzionalmente, ad un pubblico mondiale, che vuole parlare a tutti (ed è superfluo sottolineare quanto di manzoniano vi sia in questa idea): non solo attraverso l'uso di una lingua «media» che abolisse programmaticamente le barriere tra letteratura «colta» e letteratura popolare, ma con la volontà precisa di affidare alle proprie opere un messaggio che dalla specola di una piccola città di provincia si rivolgesse ai cittadini dell'intero mondo occidentale; in una intervista rilasciata a Roberto Bonetti per la «Voce Repubblicana» nel 1984, intitolato non a caso L'Europa della cultura e della ragione Bassani confessava infatti: «Nel romanzo di Ferrara c'è il mio messaggio all'Europa, il senso profondo del mio impegno morale e civile»; e ancora, a proposito dell'America, in cui, come si è visto, Bassani tentò con tutte le sue forze di essere conosciuto, pronunciava parole che suonano oggi inquietanti, nel loro essere quasi profetiche:

Dobbiamo vedere l'America come un esempio da correggere: è più avanti di noi sulla strada dell'industrializzazione totale, anche perché è un paese semplice, meno ricco delle infinite complessità europee. Ma è il frutto nostro, l'erede della nostra cultura e della nostra tradizione [...]. Tutti, americani ed europei, siamo nati qua, da questa parte dell'Atlantico, ma noi siamo più vicini alle radici, che sono anche loro. Difendere queste radici dalla barbarie di un mondo che considera l'uomo come un semplice oggetto da consumare, è il nostro compito comune.

\section{NOTE}

1. Cfr. I libri di Giorgio Bassani: traduzioni, tradizioni, negoziazioni (catalogo della mostra tenuta a Ferrara da novembre a gennaio 2016), a cura di R. Cupo, Bologna, Pendragon, in corso di stampa. Tutte le edizioni straniere reperite (più di duecento) sono visionabili consultando la mostra virtuale Ilibri di Giorgio Bassani nel mondo, a cura di R.Cupo, al seguente indirizzo: $<$ www.movio.beniculturali.it/unife/ilibridigiorgiobassaninelmondo/>.

2. G. Bassani, In risposta (II), in Di là dal cuore, Milano, Mondadori, 1984.

3. Lettera di Giorgio Bassani a Giulio Einaudi dell'8 gennaio 1964, Archivio storico Giulio Einaudi Editore, Archivio di Stato di Torino (d'ora in poi, Archivio storico Giulio Einaudi Editore, Torino). 4. "Se nel primo numero di "Botteghe oscure" [...] mi ripresentai narratore non fu tanto per obbedire al richiamo di una vocazione espressiva ineluttabile, [...] quanto per corrispondere all'attesa, affettuosa e imperiosa insieme, di una persona amica» (G. Bassani, Laggiù in fondo al corridoio, in L'odore del fieno, Milano, Mondadori, 1972).

5. «An Anthology of New Italian Writers», Roma, 1950, che presentava direttamente in traduzione inglese una scelta di opere già edite su «Botteghe oscure»; di Bassani (contrariamente a quanto avvenne per gli altri autori) furono pubblicate sia alcune liriche (con il titolo Poems) che un racconto, Love story; tutti i testi furono tradotti da William Weaver (Introduzione di Stefania Valli a La rivista «Botteghe oscure» e Marguerite Caetani: la corrispondenza con gli autori italiani, a cura di S. Valli, Roma, L'erma di Bretschneider, 1999, pp. 11 e 65). 
6. Lettera di Margaret Bottrall a Giorgio Bassani s.d., ma collocabile tra il settembre e l'ottobre del 1951 grazie al riferimento all'«inizio dell'anno scolastico» (Bassani aveva ottenuto un incarico al Liceo di Napoli) e all'uscita dell'VIII numero di «Botteghe oscure», Archivio Giorgio Bassani, Parigi.

7. Bassani si riferisce qui alla precedente apparizione di Love story; la stessa Bottrall, in una precedente missiva, aveva informato Bassani della «notizia dell'Antologia pubblicata in un numero recente del Times Literary Supplement, con un'allusione molto favorevole alla sua novella, Storia d'amore» (lettera del 29 maggio 1952, Archivio Giorgio Bassani, Parigi).

8. Ibid.

9. G. Bassani e M. Caetani, Sarà un bellissimo numero. Carteggio 1948-1959, a cura di M. Tortora, Roma, Edizioni di storia e letteratura, 2011, p. 127.

10. Lettera del 29 maggio 1952, Archivio Giorgio Bassani, Parigi.

11. Lettera di Ben Jonson a Bassani dell'11 maggio 1953, Archivio Giorgio Bassani, Parigi. L'antologia avrebbe dovuto includere racconti di «Alvaro, Buzzati, Soldati, Pratolini, Tecchi, Svevo, Moravia, Berto, Calvino e molti altri».

12. Lettera di Armando Vitelli a Bassani del 5 giugno 1956, Archivio Giorgio Bassani, Parigi.

13. Lettera del 26 luglio 1956 di Giulio Einaudi a Bassani, Archivio Giorgio Bassani, Parigi.

14. Lettera di Armando Vitelli a Bassani del 24 luglio 1956, Archivio Giorgio Bassani, Parigi.

15. La minaccia era tra l'altro stata più volte reiterata: «Non sperare di ingelosirci dicendo che Garzanti chissà cosa avrebbe fatto» (lettera di Armando Vitelli a Bassani del 24 luglio 1956, Archivio Giorgio Bassani, Parigi).

16. Lettera di Bassani a Foà del 5 ottobre 1956, Archivio storico Giulio Einaudi Editore, Torino.

17. Ibid.

18. Lettera di Eleanor French a G. Bassani del 3 luglio 1956.

19. Lettera di M. Caetani a G. Bassani del 18 agosto 1956, in G. Bassani e M. Caetani, Sarà un bellissimo numero, cit., p. 160.

20. Ibid.

21. Lettera della Caetani a Bassani dell's settembre 1956, in G. Bassani e M. Caetani, Sarà un bellissimo numero, cit., pp. 161-162.

22. Lettera di Bassani alla Caetani del 5 luglio 1957, in G. Bassani e M. Caetani, Sarà un bellissimo numero, cit., p. 167. Non è dato sapere per quale motivo, ma evidentemente Bassani pensava di avere ancora ottime possibilità con la Knopf, dal momento che scrive alla moglie: «L'altro giorno è arrivata la Knopf, una grande editrice americana, sessantacinquenne, che dovrebbe stampare il mio libro in America, [...] e una sua amica, del pari potente nell'industria editoriale» (lettera del 17 luglio 1957, Archivio Giorgio Bassani, Parigi).

23. Lettera di Luciano Foà a Bassani del 17 ottobre 1956, Archivio Giorgio Bassani, Parigi.

24. G. Bassani e M. Caetani, Sarà un bellissimo numero, cit., p. 171.

25. L'ALI, fondata a Torino nel 1898 da Augusto Foà, con lo scopo di favorire la diffusione degli autori stranieri in Italia, inizia ben presto a svolgere anche la funzione complementare di facilitare i contatti e gli accordi tra gli autori italiani e le case editrici estere. Dal 1946 l'ALI passa sotto la direzione di Erich Linder, destinato ad essere un personaggio di eccezionale rilievo nel panorama culturale italiano.

26. Lettera a Linder del 7 agosto 1957, Archivio dell'Agenzia Letteraria Internazionale, Fondazione Arnoldo e Alberto Mondadori, Milano (d'ora in poi Archivio ALI, Milano).

27. «Mi sono convinto che Erich Linder e i suoi agenti, nonostante la loro migliore volontà, costituiscono un serio ostacolo alla diffusione del mio libro fuori d'Italia» (lettera a Luciano Foà del 3 dicembre 1957, Archivio storico Giulio Einaudi Editore, Torino).

28. In quel momento Calvino era editor della casa editrice Einaudi e fu tra l'altro imprescindibile supporto per Bassani durante la pubblicazione delle Storie ferraresi.

29. Lettera di Bassani a Foà del 8 agosto 1958, Archivio storico Giulio Einaudi Editore, Torino. 
30. Ibid.

31. Lettera a Luciano Foà del 11 novembre 1958, Archivio storico Giulio Einaudi Editore, Torino. 32. L'informazione che Bassani comunica di aver ricevuto da Antonini nella cartolina a Calvino sopra citata, circa l'intenzione di Gallimard di pubblicare un unico volume di Storie ferraresi, era corretta: il volume riunì infatti Gli occhiali d'oro e le Cinque storie ferraresi.

33. Lettera di Bassani a Erich Linder del 14 febbraio 1961, Archivio ALI, Milano.

34. Relazione di Vincent Raynaud, L'opera di Giorgio Bassani in Gallimard, passato e presente, convegno I libri di Giorgio Bassani: traduzioni, tradizioni, negoziazioni, Ferrara 15-17 novembre 2016.

35. Come è stato messo in luce dall'interessantissima relazione del prof. Andrea Bresadola, $L a$ ricezione di Bassani nella Spagna franchista: traduzioni e censura, tenuta al convegno I libri di Giorgio Bassani: traduzioni, tradizioni, negoziazioni, Ferrara 15-17 novembre 2016.

36. Non si può poi non nutrire il dubbio che la situazione di stallo sia stata superata solo grazie all'uscita del nuovo romanzo di Bassani, Il giardino dei Finzi-Contini, vero e proprio best seller cui Gallimard non avrebbe mai voluto rinunciare.

37. Archivio storico Giulio Einaudi Editore, Torino.

38. Soltanto nel $2008 \mathrm{Il} \mathrm{Giardino} \mathrm{è} \mathrm{stato} \mathrm{pubblicato} \mathrm{in} \mathrm{Russia,} \mathrm{seguito} \mathrm{nel} 2010$ dalla prima edizione delle Cinque storie ferraresi, entrambe edite dalla casa editrice Text.

39. Ora edita in G. Bassani, In risposta (II), cit.

\section{RIASSUNTI}

Il saggio ripercorre le fasi del successo editoriale delle opere di Giorgio Bassani nel mondo; attraverso l'analisi dei carteggi intrattenuti con editori e traduttori, mette in rilievo da un lato la ferrea volontà dell'autore di far giungere il proprio messaggio intellettuale e morale ad un pubblico il più ampio possibile; dall'altra la cura scrupolosa esercitata nel seguire la delicata operazione di traduzione, al fine di consegnare una versione aderente non solo ai contenuti ma anche e soprattutto alla forma del testo.

This paper reconstructs the international circulation of Bassani's œuvre. The analysis of Bassani's correspondence with publishers and translators shows his strong determination to convey his intellectual and moral message to a wide international audience, as well as the scrupulous attention he devoted to the translation process. In his supervision of a translation, Bassani's consistent goal was to develop a version that was precise, aesthetically valid, and respectful of the formal qualities of the original text.

L'essai suit les étapes du succès éditorial des œuvres de Giorgio Bassani dans le monde. L'analyse des correspondances entre l'auteur, ses éditeurs et ses traducteurs montre non seulement la volonté de Bassani de faire parvenir son message intellectuel et moral à un public aussi large que possible, mais aussi la méticulosité avec laquelle il a suivi le travail de la traduction, dans le but d'obtenir un texte non seulement exact mais aussi respectueux de la forme de l'original et de ses qualités esthétiques. 
INDICE

Mots-clés : traduction, édition, distribution, correspondances, travail éditorial

Parole chiave : operazione editoriale, diffusione editoriale, traduzione, carteggi, traduttore

Keywords : edition, editorial work, translation, correspondence of writers, translator

\section{AUTORE}

ROSY CUPO

Università degli Studi di Ferrara 